\title{
Germ cells, origin of somatic stem cells?
}

\author{
Karim Nayernia ${ }^{1}$ \\ ${ }^{1}$ North East Institute of Stem Cell Biology, Institute of Human genetics, International Centre for Life, Central Parkway, University \\ of Newcastle upon Tyne, UK
}

Germ cells are highly specialized cells that form gametes, and they are the only cells within an organism that contribute genes to offspring. Germline stem cells (GSCs) sustain gamete production, both oogenesis (egg production) and spermatogenesis (sperm production), in many organisms. Since the genetic information contained within germ cells is passed from generation to generation, the germ line is often referred to as immortal. Therefore, it is possible that germ cells possess unique strategies to protect and transmit the genetic information contained within them indefinitely. On the other hand, it was shown that germ cells are pluripotent in all stages of development. We and other groups succeeded in the long-term culture of spermatogonial stem cells (SSCs) of mice. The cells were phenotypically similar to the ES/embryonic germ cells except for their genomic imprinting pattern. They differentiated into various types of somatic cells in vitro under the conditions used to induce the differentiation of the ES cells, and the SSCs formed germline chimeras when injected into blastocysts. Furthermore, we have shown that somatic stem cells are able to differentiate to germ cells. Derivation of both male and female gametes in vitro raises the possibility of using these gametes to gain a better understanding of basic reproductive biology and, in particular, to extend the potential for therapeutic cloning, transgenic technologies and the treatment of infertility. We started three different approaches for establishment of the in vitro gametogenesis systems: 1) ES-derived gametogenesis, 2) derivation of germ cells from somatic stem cells and 3) in vitro gametogenesis based on cultured spermatogonial stem cells. We developed a strategy for the establishment of germline stem cell lines from embryonic stem cells. These cells are able to undergo meiosis, generate haploid male gametes in vitro and are functional, as shown by fertilization after intra-cytoplasmic injection into mouse oocytes. Molecular and cellular mechanisms underlying differentiation of ES to functional gametes should be elucidated in future research. In other approach, we show that bone marrow stem (BMS) cells are able to trans-differentiate into male germ cells. BMS cell-derived germ cells expressed the known molecular markers of primordial germ cells. Based on these data and published data from different group, we will discuss these data and present a hypothesis that some somatic stem cells could be direct descendants of the germ lineage. The germ lineage in order to pass genes on to the next generations creates soma and thus becomes a 'mother lineage' for all somatic stem cell lineages present in the adult body.

Keywords: germline stem cells, germ cells, spermatogonial stem cells, pluripotency

Cell Research (2008) 18:s26. doi: 10.1038/cr.2008.116; published online 4 August 2008

Correspondence: Karim Nayernia

E-mail: karim.nayernia@ncl.ac.uk

Karim Nayernia, MSc/PhD, is Chairman of stem cell biology at the University of Newcastle upon Tyne in UK. Dr Nayernia received a PhD in molecular genetics from the Medical faculty of the University of Goettingen/Germany. He got his associate professorship (Habilitation) in molecular human genetics from the Medical Faculty from the University of Goettingen in Germany. In June 2006 he moved from Germany to UK. $\mathrm{He}$ is currently professor of stem cell biology at the Institute of Human Genetics and North East England Stem Cell Institute. Dr Nayernia's research focus is germline stem cells. His lab was the first lab where derived embryonic stem cell like cells from adult germline stem cells and derived the first functional sperm cells from embryonic stem cells. Please visit his lab Webpage for more details. http://www.ncl.ac.uk/ihg/staff/ profile/karim.nayernia 